\title{
Research Paper: The Effect of Brain Teaser Games on the Attention of Players Based on Hormonal and Brain Signals Changes
}

\author{
Hamed Aliyari ${ }^{1}$ (D), Hedayat Sahraei ${ }^{2}$ (D), Sahar Golabi ${ }^{3}$ (D), Masoomeh Kazemi2 ${ }^{* *}$ (D), Mohammad Reza Daliri ${ }^{4}$ (D), Behrouz Minaei-Bidgoli B $^{5}$
}

1. Center for Human-Engaged Computing, Kochi University of Technology, Kochi, Japan.

2. Neuroscience Research Center, Baqiyatallah University of Medical Sciences, Tehran, Iran.

3. Department of Medical Physiology, School of Medicine, Abadan University of Medical Sciences, Abadan, Iran.

4. Department of Electrical Engineering, School of Electrical Engineering, University of Science and Technology, Tehran, Iran.

5. Departmentl of Computer Engineering, School of Computer Engineering, University of Science and Technology, Tehran, Iran.

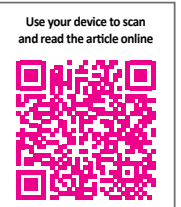

dtration: Aliyari, H., Sahraei, H., Golabi, S., Kazemi, M., Daliri, M. R., \& Minaei-Bidgoli, B. (2021). The Effect of Brain Teaser Games on the Attention of Players Based on Hormonal and Brain Signals Changes. Basic and Clinical Neuroscience, 12(5), 587-596. http://dx.doi.org/10.32598/bcn.2021.724.9

http://dx.doi.org/10.32598/bcn.2021.724.9

\section{(i) (3)}

Article info:

Received: 21 Oct 2019

First Revision: 21 Jun 2020

Accepted: 02 Aug 2021

Available Online: 01 Sep 2021

Keywords:

Brain teaser, EEG, Stress, Cortisol, $\alpha$-Amylase, Attention

\begin{abstract}
AB S T RAC T
Introduction: Computer games as an interactive media play a significant role in the cognitive and behavioral health of the players. Computer games have either positive or negative effects on cognitive indices among players. They also directly influence the lifestyle and quality of life of children, adolescents, and young adults. The present study aimed to evaluate the short-term effects of the brain teaser game on players.
\end{abstract}

Methods: Among 45 male volunteers, 40 subjects with an average age of 20 years were recruited and divided into two groups: the experimental group and the control group. All required tests were conducted before and after the intervention (playing the game) on the experimental group. Also, the same tests were performed on the control group, in which the participants were not allowed to play the game. All participants completed a questionnaire comprised demographic characteristics and specific information regarding the game (e.g., game style and hours spent on playing the game). The saliva samples were collected to measure levels of cortisol and $\alpha$-amylase. The salivary $\alpha$-amylase (sAA) and cortisol levels were analyzed using the relevant ELISA kits. The cognitive tests were performed using PASAT software before and after the game to assess the perceptual-cognitive abilities of the players. The brain waveforms were acquired by a 14-channel Emotiv brain signal recording device before and after the game. Data analysis was conducted in R and MATLAB software.

Results: PASAT test suggested that mental health and sustained attention were significantly improved after the intervention. In addition, the sAA and salivary cortisol levels were significantly higher before the intervention. The results of the brainwave analysis revealed that stress index and attention were significantly higher before the intervention.

Conclusion: Findings of the present study suggest that brain teaser games positively influence the central nervous system and activate stress path, leading to changes in brain signals and subsequently improved cognitive elements, such as attention among players.

\section{* Corresponding Author:}




\section{Highlights}

- Mental health and sustained attention were significantly improved after playing the brain teaser game.

- The salivary $\alpha$-amylase and cortisol levels were significantly higher after the brain teaser game.

- Stress and attention were significantly higher after the brain teaser game.

\section{Plain Language Summary}

The widespread use of video games with their positive or negative cognitive effects on the players has highlighted the need for research in this area. The positive cognitive effects of video games include improved cognitive indices and increased mental flexibility of the players. The present study was carried out to examine the impact of brain teaser video games on the central nervous system of gamers. Our research shows that logic stress and limit stress generated during playing brain teaser games are positive stress, with a desirable effect on the players' attention. These two types of stress improve cognitive abilities, such as attention, concentration, and problem-solving, by activating the brain's frontal lobe. We suggest that further research be conducted to evaluate different game styles to identify stress types that positively impact cognitive abilities.

\section{Introduction}

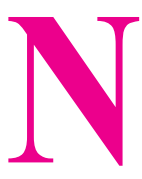

owadays, computer games have significantly influenced the Quality of Life (QoL) of children and adolescents. Also, players spent more time on video games. There is evidence that computer games significantly affect the cognitive skills and behavioral health of gamers. However, they may have adverse effects on the gamers and result in mental and behavioral disorders. The increasing number of computer games with different contents and the increased number of engaged gamers reflect the importance of research in this area. Computer games may have positive or negative effects on cognitive indices, as they may have neurological, psychological, and physiological effects on the health of the gamers (Baranowski, Buday, Thompson, \& Baranowski, 2008; Biddle \& Asare, 2011; Ebbeling, Pawlak, \& Ludwig, 2002). According to the literature, computer games have short-term effects on the QoL, attention, and concentration of children (Tahiroglu et al., 2010).

Also, computer games and their induced stress is a major cognitive indicator that may either negatively or positively affect other cognitive indices among players (Aliyari, et al., 2018; Chan \& Rabinowitz, 2006). The results of previous studies show that the impact of stress varies depending on the playing style and content of the games. Therefore, stress plays a superb role in the strengthening or weakening of cognitive elements (Aliyari et al., 2018; Aliyari et al., 2020). According to the literature, computer games players show different types of stress, including logic stress, limit stress, and fear stress. Logic stress is mostly observed in puzzle games under which the person has no losing or time-limited stress. Puzzle games enhance the function of the prefrontal cortex, which plays an important role in cognitive abilities, such as thinking, decision making, concentration, and problem-solving. Limit stress is mostly seen in runner games under which the person is afraid of losing. The fear of losing activates the Hypothalamic-PituitaryAdrenal (HPA) axis, increasing cortisol and $\alpha$-amylase, leading to time stress. Time stress considerably improves attention, concentration, response speed, and hand-eye coordination among players (Aliyari et al., 2018; Aliyari et al., 2020). Computer games have various impacts on the secretion of cortisol, depending on the content of the game. Computer games stimulate the adrenal sympathetic system and activate the stress system (Biddle \& Asare, 2011). The impact of computer games on stress systems may be associated with many biological changes. Longterm stimulation of the HPA axis is associated with the development of many psychiatric disorders. For example, stress is linked with the HPA axis activation. Hypothalamus and brainstem directly control the stress responses. In the HPA axis, the corticotropic-releasing hormone is produced in the hypothalamus and delivered to the anterior pituitary, which releases the adrenocorticotropic hormone into the systematic circulation. The adrenocorticotropic hormone is delivered to the adrenal cortex and regulates cortisol secretion (Chan \& Rabinowitz, 2006). Stress responses may cause behavioral, autonomic, and endocrine changes in the brain (Aliyari et al., 2015). Also, activation of the stress system causes reactions generally called fear. These reactions activate the sympatric of the stress system, 
leading to activation of the sympathetic or parasympathetic nervous system among players (Aliyari et al., 2015; Biddle \& Asare, 2011). Computer games have several neurological effects on the central nervous system based on the content, style, and activation of the fear-stress system (Aliyari et al., 2015; Aliyari et al., 2018). It should be noted that any environmental factor that causes abnormal secretion of cortisol may affect brain signals, resulting in changes in cognitive indicators (Aliyari et al., 2019). The Electroencephalogram (EEG) measures the existing currents of the neurons in the cerebral cortex. EEG signals create an electric field across the scalp or around the head and represent the brain's electrical activity. The information regarding brain function obtained from EEG has been widely used in many fields, including medicine, research, and Brain-Computer Interface (BCI) systems over the past two decades (Shih, Krusienski, \& Wolpaw, 2012). If a device can differentiate and decode different cognitive indices of recorded mental activity while playing computer games, those activities form a simple alphabet that a person can perform a combination of different cognitive abilities to communicate with the surrounding world. Brain signal decoding, also known as brain decoding, is a well-known method to assess cognitive indicators (Shirer, Ryali, Rykhlevskaia, Menon, \& Greicius, 2012). If we consider brain signals as brain language, accurate recording of these electric signals and data processing could help extract useful data, and comparison of the results of data processing can be useful in decoding human brain signals with high accuracy (Haynes, 2009; Shirer et al., 2012). Brain wave recording test is a complicated method that demonstrates the mechanical, electrical, and biochemical activity of the nervous system. Brain wave recording test is the only non-aggressive method to display and measure functional features of the nervous system (Bersak et al., 2001; Shim, Lee, \& Shin, 2007). Previous studies have examined the average level of stress and arousal from brain wave analysis (Aliyari et al., 2018; Bersak et al., 2001; Biddle \& Asare, 2011). Many psychiatric and mental disorders resulting from playing computer games can be prevented if the pathological use of computer games and related health impacts are investigated based on the content and style of the computer games.

The present study evaluated the neurological effects of brain teaser games on players and examined whether the neurological indicators improve or decline after playing the brain teaser games. We also determined the type of stress in playing brain teaser games and the positive or negative effects of stress on players. Also, we assessed whether a specific cognitive indicator is improved after playing a brain teaser game. We performed cognitive and biological tests and brain wave analysis to achieve these objectives.

\section{Methods}

Among 45 male volunteers, a total of 40 subjects with an average age of 20 years were recruited and divided into two groups: the experimental group $(\mathrm{n}=20)$ and the control group $(\mathrm{n}=20)$. All required tests were conducted before and after the intervention (playing the game) on the experimental group. Also, the same tests were performed on the control group in which the participants were not allowed to play the game. All subjects were screened for eligibility, and the inclusion criteria were no history of playing the assigned game, no illicit drug use, no specific medical condition, and no medication use. All assigned subjects completed a questionnaire comprised demographic characteristics and specific information regarding the game (e.g., game style and hours spent on playing the game). Then, PASAT software was used to assess the mental health and sustained attention of the players. All participants were trained to perform the test correctly. The assigned game was a brain teaser. The PASAT was performed before and after the intervention in the experimental group and the control group without the intervention. The results were analyzed using R software.

Before and after the intervention, saliva samples were collected from all participants in 10-mL falcon tubes and were maintained at $-20^{\circ} \mathrm{C}$ in the fridge to compare the cortisol and $\alpha$-amylase levels. On the experiment day, the samples were melted at room temperature, and after centrifuging at $3000 \mathrm{~g}$ for 5 minutes, $20 \mu \mathrm{L}$ of each sample was separated for testing. Then, the cortisol ELISA kit was used to measure human salivary cortisol (Cortisol ELISA KIT, Diagnostics Biochem Canada Inc, dbc) and a specific salivary kit ( $\alpha$-Amylase ELISA KIT, Pars azmoon Inc) for the measurement of sAA. The obtained data were analyzed in statistical $R$ software.

\section{Brainwave signal analysis}

The following procedure was conducted to extract the required data from the brain function of the individuals and an acceptable level of generalization ability.

First, a standard and measurable stimulant was applied to stimulate each cognitive element. Data were labeled based on the degree of stimulation (measurable metrics to provide quantitative feedback regarding impacts). If there was no standard stimulant for a specific cognitive element, an independent stimulant was searched for each particular cognitive element, such as a stress-inducing scene in a movie. Then, the recognition reference of each cognitive element was standardized (e.g., the sali- 
vary cortisol was measured to examine the effect of an assigned stimulus on stress, self-report in specific tests, or application of more accurate indicators). Afterward, the person was positioned in a basic condition without any triggering stimuli, and brainwave signals were recorded. Then, for each cognitive element, the person was exposed to the stimulus for a controlled duration without awareness of the stimulus. The recorded brain signals during this period were labeled as stimulus-trigger.

After brain signals were obtained and recorded from all participants at both basic and stimulus-triggered conditions, all brainwave signals were evaluated to extract brain signal patterns for each cognitive stimulus. Accordingly, after removing eye blink artifacts and redundant energy sources through Fourier transform, we divided the signals of each electrode into different frequency bands. In the next stage, the energy of bands was calculated in each specific electrode and frequency band. Then, frequency bands and electrodes with significant increase or decrease in signal power between brain signals of individuals were highlighted. Significance of changes is a relative concept that is characterized by minimum or maximum rates of change.

The selected frequency band is the best indicator of cognitive stimulus triggered condition on a person and is called "primary indicator". In the next stage, the existing primary indicator was searched in the study population, and after generalization, the rate of the primary indicator was shown to decrease. In other words, we found the frequency bands and locations that effectively contribute to identifying specific cognitive elements known as "secondary indicators".

The starting point of this algorithm has a significant effect on obtaining primary and secondary indicators. Therefore, we use an over-innovative algorithm to start and navigate the statistical community to complete this process. At a closer look, extracting the primary indicators and the order of generalization of these indicators may play a significant role in secondary indicators.

Eventually, to increase accuracy, the reference standard tags were placed on a person's brain signals to determine the extent to which the impulses affected brain signals in each person and whether changes in the power of frequency bands in the primary and secondary cognitive indicators were consistent with reference standard changes or not.

If the answer is yes, the extraction pattern for the specific cognitive element yielded satisfactory results. Otherwise, by applying a finite factor for that frequency band with a particular position (reducing the effect of that primary or secondary cognitive indicator), we move to the start point of the path and try to extract a new pattern.

The EEG signals were recorded using a 14-channel EEG-Emotive device. For this purpose, all electrodes were placed on specific regions of the head (Figure 1). Then, the brainwaves were recorded with closed and opened eyes. EEG signals were recorded before, during, and after the intervention by the EEG-Emotive device. EEG signals were also recorded in the control group without the intervention. Brainwave signals were analyzed using MATLAB software. To extract features, we calculated and compared the energy of signals in different bands and electrodes. Data obtained from brainwave analysis are helpful to measure players' attention and stress (Aliyari et al., 2018; Aliyari et al., 2019; Bleakley et al., 2015; Shim, Lee, \& Shin, 2007).

All clinical and lab researchers employ the $\theta$ band in the Frontal Cortex (FC) region and the ratio of $\frac{\theta}{\beta}$ to measure attention. The increased energy of $\theta$ in the $\mathrm{FC}$ region and the ratio of $\frac{\theta}{\beta}$ is associated with decreased attention.

$$
\text { Attention }=\frac{\text { Pow }\left(\mathrm{AF}_{\text {beta }}\right)+\text { Pow }\left(\mathrm{AF}_{\text {beta }}\right)}{\text { Pow }\left(\mathrm{AF} 3_{\text {theta }}\right)+\text { Pow }\left(\mathrm{AF} 4_{\text {theta }}\right)}
$$

, where pow $(\mathrm{Ab})$ is the signal power of electrode $\mathrm{A}$ in band $b$.

According to previous studies, the activity of the right hemisphere is higher relative to the left in people with social stress and anxiety or those exposed to social threats. Since brainwave analysis explains the ratio of the activity of the right hemisphere relative to the left

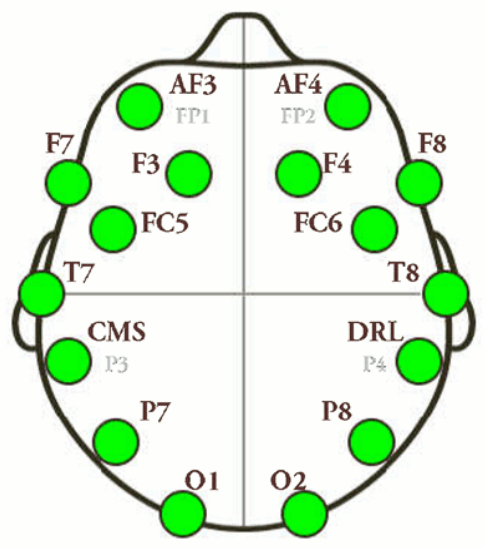

NEUR SCIENCE

Figure 1. A schematic location of the electrodes on the heads of the participants 


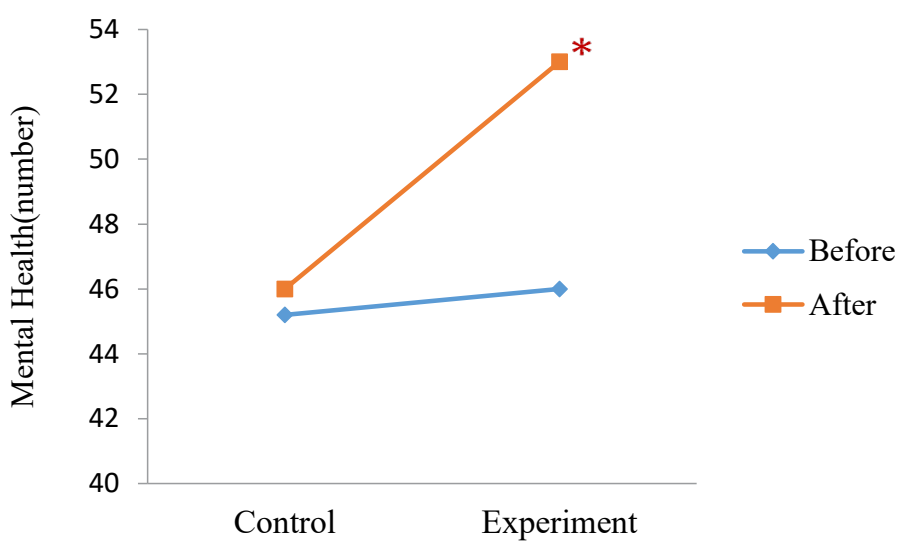

NEUR:SCIENCE

Figure 2. Changes in mental health of the participants in the experimental and control groups comparing mental health among participants before and after the intervention

$* \mathrm{P} \leq 0.05$.

hemisphere, it can be effectively used to determine the level of stress among individuals (Aliyari et al., 2019; Aliyari et al., 2019).

\section{Statistical analysis}

The data were expressed as Mean and Standard Deviation (Mean \pm SEM). The Wilcoxon signed-rank test was applied to compare the mean of the two communities. Data on brainwave functions were analyzed by MATLAB. The significance level was set at 0.05 .

\section{Results}

Results of the PASAT test show that mental health (the sum of correct answers) and sustained attention (the longest chain of subsequent correct responses) were sig- nificantly improved after the intervention. However, no significant difference was observed in the control group (Figures 2 and 3). In addition, biological tests revealed that the sAA and salivary cortisol levels were significantly higher after the intervention. However, there was no significant change in the control group (Figures 4 and 5). The results of the brainwave analysis revealed that stress and attention were significantly higher after the intervention. Nevertheless, no changes were observed in the control group (Figures 6 and 7).

\section{Discussion}

Nowadays, computer games play a key role in the QoL of players. Hence, there is a growing number of studies about computer games and their negative and posi-

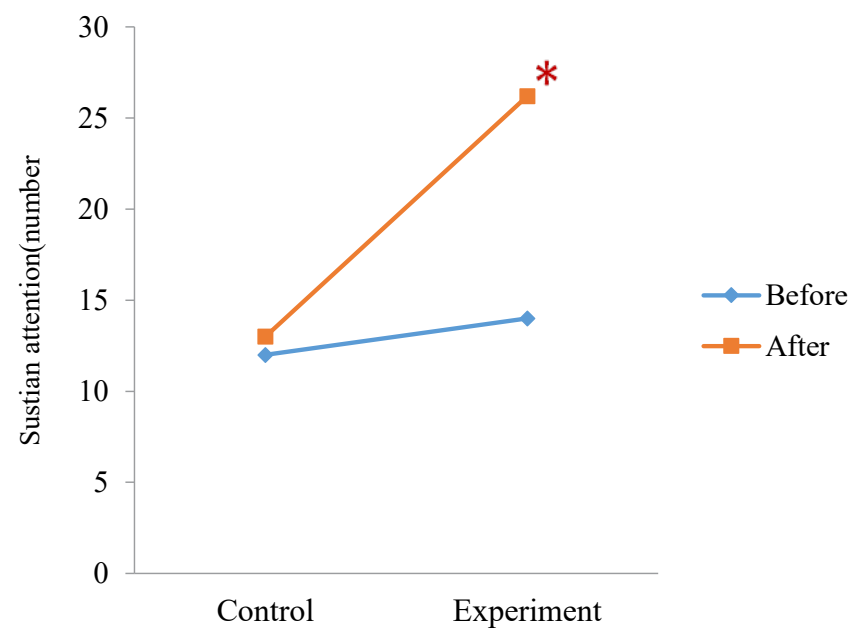

Figure 3. Changes in sustained attention in the experimental and control groups 


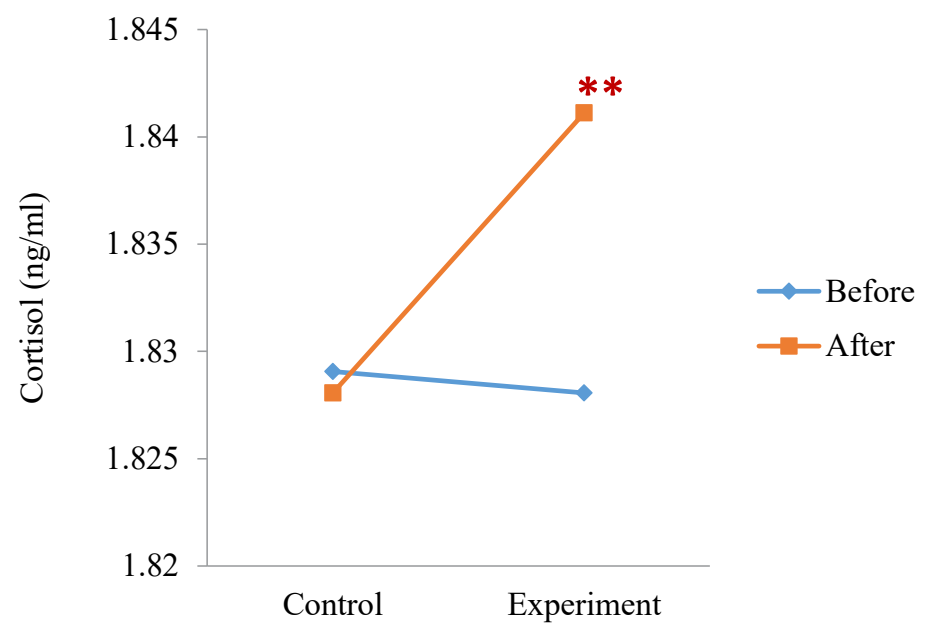

Figure 4. Changes in salivary cortisol in the experimental and control groups

NEUR SCIENCE

Comparing cortisol levels among participants before and after the intervention; ** $\mathrm{P} \leq 0.01$.

tive effects. The content and style of some games have been shown to affect mental and cognitive health, and even the physical health of the individuals (Baranowski et al. 2008; Bleakley et al., 2015; Papastergiou, 2009; Tahiroglu et al., 2010). Nowadays, various studies have been done on the effects of computer games on the Central Nervous System (CNS) of the players. Computer games as an environmental factor cause gene expression, hormonal, neurological, and behavioral changes in CNS. According to the literature, environmental factors may adversely affect the neurological system, normal secretion of hormones, and even gene expression of $\mathrm{N}$ methyl-D-aspartate (NMDA) receptors (Sadat-Shirazi et al., 2018; Tekieh et al., 2017). The gene expression of NMDA receptors plays a key role in developing cognitive abilities such as attention, concentration, memory, and learning among animals and human beings. Envi- ronmental factors in urban areas, such as electromagnetic fields, electric fields, and even computer games, act as activators or inhibitors of gene expression of NMDA receptors (Aliyari et al., 2019; Kazemi et al., 2018; Sadat-Shirazi et al., 2018). Several studies have shown that computer game addiction would alter the expression level of NMDA receptors (Sadat-Shirazi et al., 2018). Computer games have either positive or negative effects on the players' cognitive abilities based on the game content and style. In other words, the nervous system of the players is entirely influenced by computer games (Tahiroglu et al., 2010). Cognitive tests conducted by PASAT revealed that mental health and sustained attention were improved after playing brain teaser games, which is supported by the results of cognitive tests on mobile runner games. However, there was no significant difference in cognitive abilities and mental

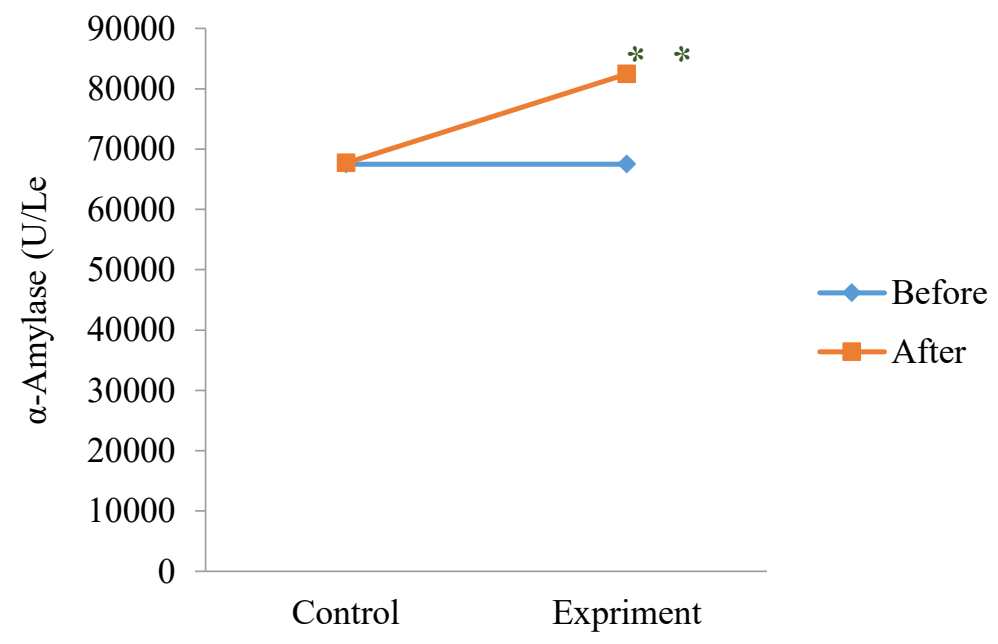

Figure 5. Changes in a-amylase in the experimental and control groups

Comparing a-amylase levels among participants before and after the intervention; ** $\mathrm{P} \leq 0.01$. 


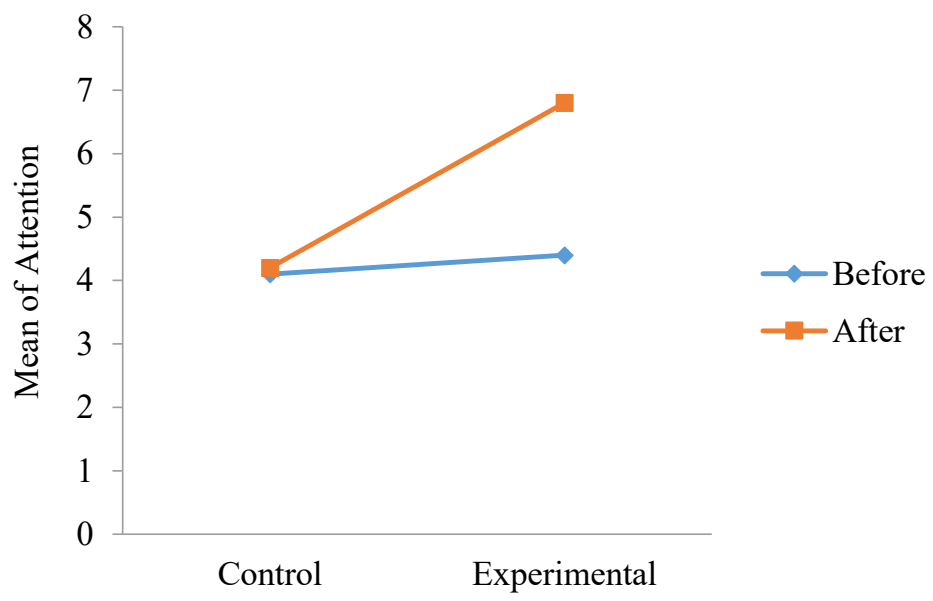

Figure 6. Changes in attention in the experimental and control groups

Mean changes in the power of stable brainwaves energy, construct of attention among players.

health in the control group. Moreover, biological tests revealed that the salivary cortisol and sAA levels were higher, after playing brain teaser game. Similarly, previous studies showed that the secretion of salivary cortisol and sAA increased, after playing runner games. The type of stress produced during playing runner games is a limit stress, which is mostly due to fear of losing in these types of games. Therefore, limit stress has been shown to be critically associated with attention, concentration, and response speed. Similar to runner games, the type of stress in brain teaser games is limit stress. However, unlike runner games, the limit stress in brain teaser game is mostly attributed to time limits. Levels of salivary cortisol and sAA were not significantly different in the control group. Attractive colors and time limits in brain teaser game improved attention and concentration of the players after the intervention. In fact, the assigned game in this study similar to puzzle games improved brain function, especially frontal lobe. Thus, in addition to limit stress, another type of stress called "logic stress" is seen after playing brain teaser games (Anderson et al., 2002; Lee \& Faber, 2007). Cortisol has various impacts on the human and animals body at different dosages. Accordingly, any stimulus like environmental stimulus may cause abnormal cortisol secretion, leading to the appearance of different cognitive behaviors. Environmental factors affect cortisol secretion by influencing the HPA axis and adrenal cortex (Lupien, McEwen, Gunnar, \& Heim, 2009). Environmental inhibitors of the nervous system reduce the normal secretion of cortisol, resulting in psychiatric disorders such as depression among both human and animals. However, environmental stimulus of nervous system increases the normal secretion of cortisol leading to cognitive disorders such as violence, anxiety, and reduced attention and concentration in human beings and animals (Aliyari et al., 2018; Aliyari et al.,

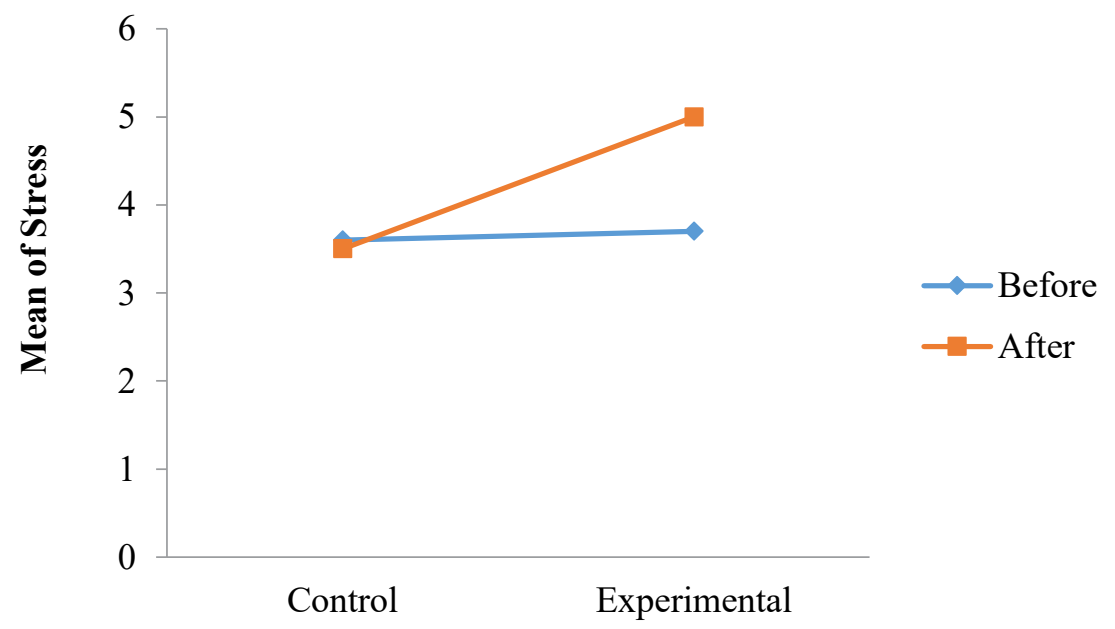

Figure 7. Changes in stress level in the experimental and control groups 
2020; Aliyari et al., 2019; Kazemi et al., 2017; Lupien, Maheu, Tu, Fiocco, \& Schramek, 2007). Since cortisol is a dose-dependent hormone, it plays a critical role in the processing of learning and memory formation in humans and animals (Aliyari et al., 2019; Kazemi et al., 2018; Kazemi et al., 2018). Several studies have shown that playing piano games significantly reduced the progression of Alzheimer disease. Furthermore, the rhythm of the piano plays an important role in learning piano for piano learners (Robert et al., 2014). Computer games as an environmental stimulus of the stress-fear system may have various behavioral, mental, or neurological effects, depending on the personal characteristics of the individuals. Identification of the type and degree to which the content and style of computer games affect the nervous system of players may contribute to either strengthening or weakening the cognitive indicators of the players (Baranowski et al., 2008; Biddle \& Asare, 2011; Tahiroglu et al., 2010; Tekieh et al., 2017).

Cortisol is a stress marker, and $\alpha$-amylase is a fear marker. These two markers may have either negative or positive effects on the fear-stress system. The positive effects are associated with improved neurological indicators, while adverse effects are associated with reduced positive cognitive function and increased negative cognitive function (Aliyari et al., 2018; Kazemi et al., 2018).

Brainwave signal tests such as EEG is a common method to measure cognitive load online. Processing and analysis of brain wave signals provide helpful information regarding different characteristics of the person. The range of EEG signals depends on the concurrency of the cerebral neurons (Badcock et al., 2013). Concurrent stimulation of a group of neurons may lead to producing a high amplitude signal on the skin because the signal generated from each neuron gathers together in a timedomain (Rodrak \& Wongsawat, 2013). The frequency of these concurrent stimuli also results in the formation of a periodic EEG signal at a given frequency. Also, the identification of particular brain bands may help strengthen or weaken the desired brain band. These signals contain helpful information regarding the function of different parts of the brain (Badcock et al., 2013).

Brain signal analysis identifies various brain wave locations based on the severity and weakness of the location. Hence, brain wave analysis can be widely utilized in mind engineering, extraction of cognitive indicators, and recognition of various pathological conditions, such as epilepsy, brain tumors, and sleep disorders (Badcock et al., 2015; Liu, Chiang, \& Chu, 2013; Mahajan \& Morshed, 2015; Sanei \& Chambers, 2007; Wang,
Jung, Chen, Huang, \& Lin, 2013). There is evidence that brain signals change after playing computer games based on the content and style of the game (Badcock et al., 2013; Ramirez \& Vamvakousis, 2012). The present study's findings show that the stable brain signal powers for stress and attention were higher after playing a brain teaser game. However, brain signal analysis showed no significant difference in the control group. Results of biological tests and brain signal analysis revealed that stress increased after the intervention. Also, both logic stress and limit stress appeared while playing the brain teaser game. Both types of stress positively affect the frontal lobe's function, which explains the improved attention and concentration after playing a brain teaser game. Moreover, the PASAT test revealed that attention and the mean brain signal power increased after playing a brain teaser game. Thus, both logic stress and limit stress generated while playing brain teaser games are positive stresses and positively affect players' attention. These two types of stress improve cognitive abilities, such as attention, concentration, and problem-solving, through activation of the brain's frontal lobe. We suggest that further research be conducted to evaluate different game styles to identify stress types that positively impact cognitive abilities.

\section{Ethical Considerations}

\section{Compliance with ethical guidelines}

All experiments were approved by the Ethics Committee of Neuroscience Research Center of Baqiyatallah University of Medical Sciences (Code: IR.BMSU.1394.112).

\section{Funding}

The study was financially supported by Soft Technology Development Council and Neuroscience Research Center of Baqiyatallah University.

\section{Authors' contributions}

Conceptualization: Hamed Aliyari, Mohammad Reza Daliri, and Behrouz Minaei-Bidgoli; Methodology: Hamed Aliyari, Masoomeh Kazemi, and Sahar Golabi; Investigation: Masoomeh Kazemi, Sahar Golabi, and Hamed Aliyari; Writing the original draft: Masoomeh Kazemi and Hamed Aliyari; Writing, review, and editing: Hedayat Sahraei and Mohammad Reza Daliri; Resources: Hedayat Sahraei, Mohammad Reza Daliri, and Behrouz Minaei-Bidgoli; Supervision: Mohammad Reza Daliri. 


\section{Conflict of interest}

The authors declared no conflict of interest.

\section{Acknowledgments}

We express our gratitude to the Neuroscience Research Center of Baqiyatallah University of Medical Sciences, and also Soft Technology Development Council and Neurogame Research group.

\section{References}

Aliyari, H., Hosseinian, S. H., Menhaj, M. B., \& Sahraei, H. (2019). Analysis of the Effects of High-Voltage Transmission Line on Human Stress and Attention Through Electroencephalography (EEG). Iranian Journal of Science and Technology, Transactions of Electrical Engineering, 43(Suppl 1), 211-8. [DOI:10.1007/s40998-018-0151-8

Aliyari, H., Hosseinian, S. H., Sahraei, H., \& Menhaj, M. B. (2019). Effect of proximity to high-voltage fields: Results of the neural network model and experimental model with macaques. International Journal of Environmental Science and Technology, 16(8), 4315-26. [DOI:10.1007/s13762-018-1830-8]

Aliyari, H., Kazemi, M., Tekieh, E., Salehi, M., Sahraei, H., \& Daliri, M. R., et al. (2015). The effects of Fifa 2015 computer games on changes in cognitive, hormonal and brain waves functions of young men volunteers. Basic and Clinical Neuroscience, 6(3), 193-201. [PMID] [PMCID]

Aliyari, H., Sahraei, H., Daliri, M. R., Minaei-Bidgoli, B., Kazemi, M., \& Agaei, H., et al. (2018c). The beneficial or harmful effects of computer game stress on cognitive functions of players. Basic and Clinical Neuroscience, 9(3), 177-86. [DOI:10.29252/ nirp.ben.9.3.177] [PMID] [PMCID]

Aliyari, H., Sahraei, H., Erfani, M., Mohammadi, M., Kazemi, M., \& Daliri, M. R., et al. (2020). Changes in cognitive functions following violent and football video games in young male volunteers by studying brain waves. Basic and Clinical Neuroscience, 11(3), 279-88. [DOI:10.32598/bcn.9.10.335] [PMID] [PMCID]

Anderson, P., Bell, T. A., Casey, M. P., Jaffe, J. R., Ledesma, L. R., \& Mastropietro, M. W., et al. (2002). Gaming machine with interlinked arrangements of puzzle elements. Retrieved from https:/ / patents.justia.com/patent/6428412

Badcock, N. A., Mousikou, P., Mahajan, Y., de Lissa, P., Thie, J., \& McArthur, G. (2013). Validation of the Emotiv EPOC® EEG gaming system for measuring research quality auditory ERPs. Peer], 1, e38. [DOI:10.7717/peeri.38] [PMID] [PMCID]

Badcock, N. A., Preece, K. A., de Wit, B., Glenn, K., Fieder, N., \& Thie, J., et al. (2015). Validation of the Emotiv EPOC EEG system for research quality auditory event-related potentials in children. PeerJ, 3, e907. [DOI:10.7717/peerj.907] [PMID] [PMCID]
Baranowski, T., Buday, R., Thompson, D. I., \& Baranowski, J. (2008). Playing for real: Video games and stories for health-related behavior change. American Journal of Preventive Medicine 34(1), 74-82.E10. [DOI:10.1016/j.amepre.2007.09.027] [PMID] [PMCID]

Bersak, D., McDarby, G., Augenblick, N., McDarby, P., McDonnell, D., \& McDonald, B., et al. (2001). Intelligent biofeedback using an immersive competitive environment. Retrieved from https:// www.researchgate.net/publication/246492595

Biddle, S. J. H., \& Asare, M. (2011). Physical activity and mental health in children and adolescents: A review of reviews. British Journal of Sports Medicine, 45(11), 886-95. [DOI:10.1136/ bjsports-2011-090185] [PMID]

Bleakley, C. M., Charles, D., Porter-Armstrong, A., McNeill, M. D. J., McDonough, S. M., \& McCormack, B. (2015) Gaming for health: A systematic review of the physical and cognitive effects of interactive computer games in older adults. Journal of Applied Gerontology, 34(3), NP166-89. [DOI:10.1177/0733464812470747] [PMID]

Chan, P. A., \& Rabinowitz, T. (2006). A cross-sectional analysis of video games and attention deficit hyperactivity disorder symptoms in adolescents. Annals of General Psychiatry, 5, 16. [DOI:10.1186/1744-859X-5-16] [PMID] [PMCID]

Ebbeling, C. B., Pawlak, D. B., \& Ludwig, D. S. (2002). Childhood obesity: Public-health crisis, common sense cure. The Lancet 360(9331), 473-82. [DOI:10.1016/S0140-6736(02)09678-2]

Haynes, J. D. (2009). Decoding visual consciousness from human brain signals. Trends in Cognitive Sciences, 13(5), 194-202. [DOI:10.1016/j.tics.2009.02.004] [PMID]

Kazemi, M., Sahraei, H., Aliyari, H., Tekieh, E., Saberi, M., \& Tavacoli, H., et al. (2018). Effects of the extremely low frequency electromagnetic fields on NMDA-receptor gene expression and visual working memory in male rhesus macaques. Basic and Clinical Neuroscience, 9(3), 167-76. [DOI:10.29252/NIRP. BCN.9.3.167] [PMID] [PMCID]

Lee, M., \& Faber, R. J. (2007). Effects of product placement in online games on brand memory: A perspective of the limitedcapacity model of attention. Journal of Advertising, 36(4), 75-90. [DOI:10.2753/JOA0091-3367360406]

Liu, N. H., Chiang, C. Y., \& Chu, H. C. (2013). Recognizing the degree of human attention using EEG signals from mobile sensors. Sensors, 13(8), 10273-86. [DOI:10.3390/s130810273] [PMID] [PMCID]

Lupien, S. J., Maheu, F., Tu, M., Fiocco, A., \& Schramek, T. E. (2007). The effects of stress and stress hormones on human cognition: Implications for the field of brain and cognition. Brain and Cognition, 65(3), 209-37. [DOI:10.1016/j. bandc.2007.02.007] [PMID]

Lupien, S. J., McEwen, B. S., Gunnar, M. R., \& Heim, C. (2009). Effects of stress throughout the lifespan on the brain, behaviour and cognition. Nature Reviews Neuroscience, 10(6), 434-45. [DOI:10.1038/nrn2639] [PMID]

Mahajan, R., \& Morshed, B. I. (2015). Unsupervised eye blink artifact denoising of EEG data with modified multiscale sample entropy, kurtosis, and Wavelet-ICA. IEEE Journal of Biomedical and Health Informatics, 19(1), 158-65. [DOI:10.1109/ JBHI.2014.2333010] [PMID] 
Papastergiou, M. (2009). Exploring the potential of computer and video games for health and physical education: A literature review. Computers \& Education, 53(3), 603-22. [DOI:10.1016/j. compedu.2009.04.001]

Ramirez, R., \& Vamvakousis, Z. (2012). Detecting emotion from EEG signals using the emotive epoc device. In F. M. Zanzotto, S. Tsumoto, N. Taatgen \& Y. Yao (Eds.), Brain Informatics. BI 2012. Lecture notes in computer science (pp. 175-184). Vol. 7670. Berlin/Heidelberg: Springer. [DOI:10.1007/978-3-642-351396_17]

Robert, P. H., König, A., Amieva, H., Andrieu, S., Bremond, F., \& Bullock, R., et al. (2014). Recommendations for the use of Serious Games in people with Alzheimer's disease, related disorders and frailty. Frontiers in Aging Neuroscience, 6, 54. [DOI:10.3389/fnagi.2014.00054] [PMID] [PMCID]

Rodrak, S., \& Wongsawat, Y. (2013). EEG brain mapping and brain connectivity index for subtypes classification of attention deficit hyperactivity disorder children during the eyeopened period. Paper presented at 2013 35th Annual International Conference of the IEEE Engineering in Medicine and Biology Society (EMBC), Osaka, Japan, 3-7 July 2013. [DOI:10.1109/EMBC.2013.6611268] [PMID]

Sadat-Shirazi, M. S., Vousooghi, N., Alizadeh, B., Makki, S. M., Zarei, S. Z., \& Nazari, Sh., et al. (2018). Expression of NMDA receptor subunits in human blood lymphocytes: A peripheral biomarker in online computer game addiction. Journal of Behavioral Addictions, 7(2), 260-8. [DOI:10.1556/2006.7.2018.35] [PMID] [PMCID]

Sanei, S., \& Chambers, J. A. (2007). EEG signal processing. Chichester: John Wiley \& Sons Ltd. [DOI:10.1002/9780470511923]

Shih, J. J., Krusienski, D. J., \& Wolpaw, J. R. (2012). Brain-computer interfaces in medicine. Mayo Clinic Proceedings, 87(3), 268-79. [DOI:10.1016/j.mayocp.2011.12.008] [PMID] [PMCID]

Shim, B. S., Lee, S. W., \& Shin, J. H. (2007). Implementation of a 3-dimensional game for developing balanced brainwave. Paper presented at 5th ACIS International Conference on Software Engineering Research, Management \& Applications (SERA 2007), Busan, Korea (South), 20-22 August 2007. [DOI:10.1109/SERA.2007.94]

Shirer, W. R., Ryali, S., Rykhlevskaia, E., Menon, V., \& Greicius, M. D. (2012). Decoding subject-driven cognitive states with whole-brain connectivity patterns. Cerebral Cortex, 22(1), 15865. [DOI:10.1093/cercor/bhr099] [PMID] [PMCID]

Tahiroglu, A. Y., Celik, G. G., Avci, A., Seydaoglu, G., Uzel, M., \& Altunbas, H. (2010). Short-term effects of playing computer games on attention. Journal of Attention Disorders, 13(6), 66876. [DOI:10.1177/1087054709347205] [PMID]

Tekieh, E., Riahi, E., Kazemi, M., Sahraei, H., Tavakoli, H., \& Aliyary, H., et al. (2017). Role of basal stress hormones and amygdala dimensions in stress coping strategies of male rhesus monkeys in response to a hazard-reward conflict. Iranian Journal of Basic Medical Sciences, 20(8), 951-7. [DOI:10.22038/ IJBMS.2017.9120] [PMID] [PMCID]

Wang, Y. K., Jung, T. P., Chen, S. A., Huang, C. S., \& Lin, C. T. (2013) Tracking attention based on EEG spectrum. In C. Stephanidis (Ed.), HCI International 2013 - Posters' extended abstracts. HCI 2013. Communications in computer and information science (pp. 450-454). Vol. 373. Berlin/Heidelberg: Springer. [DOI:10.1007/978-3-642-39473-7_90] 